\title{
Factors Associated With Epilepsy in Children With Periventricular Leukomalacia
}

\author{
Peter Humphreys, MD, FRCP $(\mathrm{C})$, Raywat Deonandan, PhD, Sharon Whiting, MD, FRCP $(\mathrm{C})$, \\ Nick Barrowman, PhD, Mary-Ann Matzinger, MD, FRCP $(C)$, Valerie Briggs, MD, FRCP $(C)$, \\ Julie Hurteau, MD, FRCP(C), and Eda Wallace, MD
}

Children with cerebral palsy associated with periventricular leukomalacia frequently develop unprovoked epileptic seizures. This article reports an analysis of risk factors for epilepsy in children with radiologically confirmed periventricular leukomalacia. This cohort was screened for epilepsy and for an array of clinical and demographic factors. Of 154 subjects with radiologically confirmed periventricular leukomalacia, $40(26.0 \%)$ had epilepsy. In the epileptic group, radiologic pathology other than periventricular leukomalacia was uncommon. Significant associations were found between epilepsy and cerebral palsy patterns other than spastic diparesis, mental handicap, cortical visual impairment, neonatal seizures, and severe periventricular leukomalacia. Only the presence of neonatal seizures was significantly associated with epilepsy once other risk factors were controlled in the regression model. Some previous studies have shown an association between neonatal seizures and later epilepsy for cerebral palsy in general. This is the first report of such an association for a single predominant type of cerebral pathology.

Keywords: epilepsy; periventricular leukomalacia; cerebral palsy
$\mathrm{U}$ nprovoked epileptic seizures frequently develop in children with cerebral palsy. ${ }^{1-8}$ In general, the incidence of epilepsy increases with the severity of clinical involvement: rates of epilepsy are consistently higher in patients with spastic quadriparetic and hemiparetic patterns than in those with diparesis. The incidence of epilepsy in cerebral palsy also varies with the type of radiologic pathology $y^{6,7,10}$ and tends to be higher when the pathology is more severe. ${ }^{7,9}$ Epilepsy occurs in most individuals with large cortical lesions, whether unilateral or bilateral, pathologies usually associated with arm-dominant quadriparesis or hemiparesis. ${ }^{1,11,12}$

Although less common, epilepsy is nevertheless a significant complication for children with periventricular leukomalacia, with reported incidence figures of $16.0 \%$ to $47 \% .^{6,9,13}$ The main patterns of cerebral palsy in children with periventricular leukomalacia are spastic diparesis and

From the Division of Neurology, Department of Pediatrics (PH, RD, SW); the Research Institute (RD, NB), and the Department of Radiology (MAM, VB, JH, EW), Children's Hospital of Eastern Ontario; and Ottawa Children's Treatment Centre (PH), Ottawa, Ontario, Canada.

Address correspondence to: Dr Peter Humphreys, Division of Neurology, Children's Hospital of Eastern Ontario, 401 Smyth R, Ottawa, Ontario K1H8L1, Canada; e-mail: phumphreys@cheo.on.ca.

Humphreys P, Deonandan R, Whiting S, Barrowman N, Matzinger MA, Briggs, V, Hurteau J, Wallace E. Factors associated with epilepsy in children with periventricular leukomalacia. J Child Neurol. 2007;22:598-605. leg-dominant quadriparesis. ${ }^{11}$ The etiology of seizures in patients with periventricular leukomalacia is unclear, but may involve several factors acting alone or in varying combinations. Potential etiologic factors include the distribution and severity of white matter pathology, focal or diffuse cortical injury, concomitant phenomena such as intracranial hemorrhage or infection, and genetic predisposition.

To our knowledge, there is only 1 published study ${ }^{7}$ of epilepsy in cerebral palsy in which a logistic regression analysis for potential predisposing factors has been performed; this study, however, dealt with all forms of cerebral palsy. Empirically, it would be helpful to attempt to tease out predisposing factors for specific patterns of cerebral palsy pathology rather than for all types of pathology combined.

Although 2 population-based reports have included identified cohorts of periventricular leukomalacia patients, ${ }^{6,9}$ the numbers are relatively small ( 34 and 80 , respectively), and potential risk factors for epilepsy are not systematically addressed. Two additional studies ${ }^{10,14}$ specifically considered the severity of periventricular leukomalacia, but the number of subjects is even smaller (27 and 21 , respectively) and includes only those with infantile spasms. The objective of this article is to report an analysis of risk factors for epilepsy in a large population-based cohort of children with radiologically confirmed periventricular leukomalacia. 


\section{Methods}

\section{Subjects}

This study used the database at the Ottawa Children's Treatment Center, the only habilitation center for children with cerebral palsy in the geographic region of Eastern Ontario (population, 1.2 million). All regional children and adolescents with cerebral palsy are treated and followed up at this center.

In September 2000, the active client database was searched for all children with patterns of cerebral palsy known to be typically associated with bilateral periventricular leukomalacia: spastic diparesis, leg-dominant spastic quadriparesis, spastic triparesis, and spastic diparesis with dystonic or ataxic features. ${ }^{11}$ Patients with hemiparetic cerebral palsy were excluded because, although some patients with leg-dominant hemiparesis have periventricular leukomalacia, ${ }^{12}$ the pathology is typically asymmetric or even unilateral. On rare occasions, periventricular leukomalacia can be documented in individuals with other patterns of cerebral palsy (eg, arm-dominant quadriparesis, dyskinetic-dystonic quadriparesis) but is usually overshadowed by more significant pathology such as diffuse cortical atrophy, border-zone cortical/subcortical infarcts, and status marmoratus.

A retrospective chart review of all clients with appropriate patterns of cerebral palsy was performed to exclude, where possible, all those with etiologies not known to produce periventricular leukomalacia. Subjects were excluded if there was a history of a neurologic insult occurring after the postnatal period (eg, meningitis, shaken-baby syndrome), a known genetic syndrome, evidence of progressive neurologic deterioration, or a family history of a similar pattern of cerebral palsy. Also excluded were subjects whose imaging studies revealed the presence of major brain malformations such as agenesis of the corpus callosum, schizencephaly, or polymicrogyria.

\section{Clinical Data}

Data retrieval occurred after receipt of approval from the Research Ethics Board of the Children's Hospital of Eastern Ontario and the Science Committee of the Ottawa Children's Treatment Center. Clinical information was extracted for all subjects with appropriate cerebral palsy patterns regardless of whether there was radiologic evidence for the presence of periventricular leukomalacia.

Detailed historical information concerning each subject was obtained from a standardized questionnaire completed by parents at the time of their child's registration at the Children's Treatment Center. The questionnaires contained data concerning pregnancy length, number of fetuses, complications of pregnancy, delivery, birth weight, Apgar scores, neonatal course and complications, and family history. In addition, data were extracted from the
Children's Treatment Center and hospital files concerning the subject's current ambulatory status, cognitive function, visual and auditory capacity, and the presence or absence of behavioral problems.

All of the patient files were reviewed by the same neurologist $(\mathrm{PH})$. Clinical pattern and ambulatory status categorizations were based on the most recent evaluation done by a neurologist or a child development specialist. Ambulatory status was categorized as independent ambulation, walking only with the use of mechanical aids (cane, crutches, walker), or wheelchair dependence. Cognitive function was coded as "normal" (school performance appropriate for age), "learning disabled" ( 2 or more years below age level in at least 1 subject despite full-scale scores in the normal or borderline range on psychologic testing), or "mentally handicapped" (full-scale scores on psychologic testing below the borderline range). A cognitive functional level was not given if the subject was deaf or younger than 6 years old. The psychologic tests used to quantify cognitive function were, depending upon the patient's age and verbal abilities: the Wechsler Preschool and Primary Scale of Intelligence (WPPSI-R or WPPSI-III), the Wechsler Intelligence Test for Children (WISC-III or WISC-IV), the Wechsler Individual Achievement Test (WIAT or WIAT-2), and the Wide Range Assessment of Memory and Learning (WRAML or WRAML-2). Tests were administered in either English or French, depending upon the language of school instruction.

Data were extracted concerning all epileptic seizures, whether febrile or unprovoked. Subjects were classified as epileptic if, at the time of survey, they had a history of more than 1 unprovoked seizure. In all cases, the diagnosis of epilepsy was made by a pediatric neurologist.

Coded information concerning seizures included the types of seizures observed and age at onset for each seizure type. Data were also extracted for maximum seizure frequency, age of last seizure for each type, antiepileptic drugs used, response to treatment, and electroencephalograph (EEG) findings. Because these data do not pertain directly to a risk factor analysis, they are not presented in this report.

\section{Analysis of Cranial Imaging Studies}

The radiologic diagnosis of periventricular leukomalacia was confirmed on computed tomography (CT) with or without magnetic resonance imaging (MRI) studies, based on published criteria. ${ }^{15,16}$ Subjects lacking CT/MRI studies were also considered as having proven periventricular leukomalacia if typical acute changes were present on neonatal cranial ultrasound studies.

For scoring purposes, all available cranial CT and MRI studies for each subject were evaluated in a blinded fashion by 4 radiologists. Studies were graded on the severity of the periventricular leukomalacia and the presence or absence of other radiologic abnormalities according to the following scoring system: 


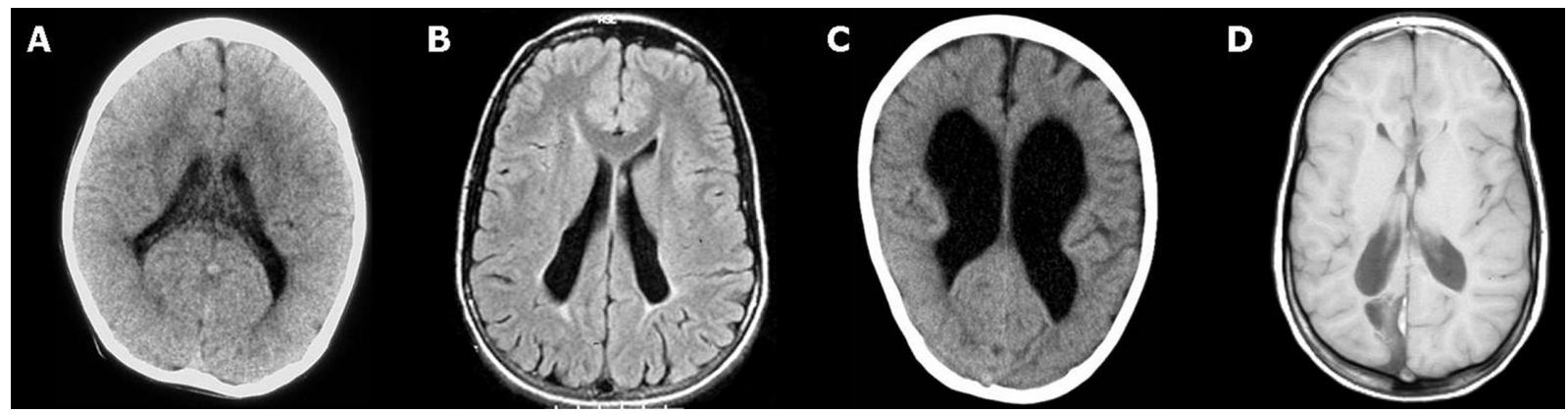

Figure 1. Examples of periventricular leukomalacia scores 1 to 4. (A) Category 1-axial computed tomography (CT), patient 9, leg-dominant quadriparesis. (B) Category 2 - axial magnetic resonance fluid-attenuated inversion recovery sequence, patient 16, diparesis. (C). Category 3-axial CT, patient 22, triparesis. (D) Category 4-axial MR, T1-weighted sequence, patient 61. In addition to demonstrating features of category 2, the image shows a right mesial occipital infarct. The anterior corpus callosum has been sectioned in a successful attempt to control medically refractory atonic seizures.

Category 0-no radiologic abnormalities; that is, no evidence of periventricular leukomalacia;

Category 1 -bilateral effacement of periventricular white matter in the posterior ventricular regions, without enlargement of the adjacent lateral ventricles;

Category 2-same white matter abnormalities as category 1 , with the addition of enlargement of the trigone and body of at least 1 lateral ventricle;

Category 3-findings as in category 2, plus effacement of periventricular white matter anteriorly and enlargement of at least 1 anterior horn;

Category 4-a periventricular leukomalacia score of 1, 2, or 3 plus at least 1 other radiologic abnormality; for example, hippocampal atrophy, focal cortical infarcts, arterial border-zone infarcts, focal cortical atrophy, porencephaly, intracerebral calcifications, thalamic or lenticular nucleus atrophy, brainstem atrophy.

Examples of periventricular leukomalacia scores 1 to 4 are shown in Figure 1. In the case of asymmetric white matter pathology, the score applied was that of the more involved cerebral hemisphere.

Given the length of time required to evaluate each imaging study and the large number of studies to be evaluated, it was deemed impractical to have 1 radiologist score all the studies. We therefore conducted an initial interobserver variability study in which 4 radiologists used the above scoring system to independently review 22 studies. A sample size of 22 studies from 22 different subjects was selected to allow for an interrater reliability of 0.8 (given 4 radiologists, $\alpha=0.05, \beta=0.2$ ).${ }^{17}$ When the level of correlation between observers was found to be good (0.78), we divided the studies to be evaluated among the 4 radiologists.

\section{Data Analysis}

Data analysis was confined to all subjects meeting the inclusion criteria who had radiologic evidence of periventricular leukomalacia (1 or more of postnatal cranial ultrasound findings, CT, and MRI). The radiologically confirmed subjects were compared with those lacking imaging studies with respect to gender, percentage born prematurely, clinical pattern of cerebral palsy, and percentage with epilepsy.

Subjects with confirmed bilateral periventricular leukomalacia were sorted into those with epilepsy and those without epilepsy. Overall differences between epileptic and nonepileptic groups were ascertained by using Pearson $\chi^{2}$, Fisher exact, and Mann Whitney $U$ tests. Univariable associations between epilepsy status and a selection of putative risk factors for epilepsy were measured by using $\chi^{2}$ tests. The risk factors examined were periventricular leukomalacia severity, clinical pattern of cerebral palsy presentation, cortical visual impairment, ambulatory status, cognitive function, history of neonatal seizure, intrauterine growth retardation, sex, bleeding during pregnancy, preterm delivery, intraventricular hemorrhaging, family history of epilepsy, and infection during pregnancy.

Those variables whose univariable analyses showed an association value of $P \leq .200$ were included in a logistic regression analysis to determine which factors, if any, were associated with epilepsy in this clinical sample. Some putative risk factors were dichotomized to allow for more robust statistical manipulations: periventricular leukomalacia severity as score categories 1 and 2 versus categories 3 and 4; clinical pattern as spastic diparesis versus all other types; ambulatory status as independent walking versus other modes of ambulation; cognitive function as mentally handicapped versus other categories.

\section{Results}

After application of the inclusion and exclusion criteria to the client database, 215 subjects were identified with appropriate patterns of cerebral palsy. Of these, 154 had 
radiologically confirmed periventricular leukomalacia, and 61 had either never had a cranial imaging study $(n=50)$ or lacked sufficient information to make such determination $(\mathrm{n}=11)$. Of those with documented periventricular leukomalacia, $40(26.0 \%)$ had a history of recurrent unprovoked epileptic seizures, and 5 other subjects reported having had singleton seizures.

Among those with epilepsy, 15 (37.5\%) of 40 had a single seizure type, and $25(62.5 \%)$ had multiple seizure types. Seizure patterns reported included generalized tonic-clonic in 19 , focal with or without secondary generalization in 17, complex partial in 17, myoclonic in 8 , tonic-adversive in 5, simple partial in 3, atypical absence in 3 , typical absence in 2, and atonic in 1. Infantile spasms developed in 7 individuals (17.5\%), 5 of whom went on to have other seizure types.

Median age at seizure onset was 30.0 months among those with epilepsy. Those with spastic diparesis had a significantly higher median age at onset (34.0 months) compared with those with other clinical patterns (median age, 15.5 months).

A comparison of the 154 subjects with confirmed periventricular leukomalacia with the other 61 subjects showed no significant differences with respect to gender or to the proportion born prematurely. As expected, the comparison group had a higher percentage of subjects with milder cerebral palsy: $83.6 \%$ spastic diparesis versus $67.2 \%$, and $11.5 \%$ leg-dominant spastic quadriparesis versus $24.2 \%$. In the comparison group, the percentage of subjects with epilepsy was much lower $(8.2 \%$ vs $26.0 \%)$, revealing a probable bias toward the performance of imaging studies in children who were epileptic or more severely involved.

\section{Risk Factor Analysis}

The analysis of putative risk factors for epilepsy is summarized in Table 1. In the univariable analysis, the epileptic group $(n=40)$ was significantly different from the nonepileptic group $(n=114)$ with respect to the distributions of levels of ambulatory status $(P=.008)$, with epileptic subjects more likely to require assistance; cognitive function $(P<.001)$, with epileptic subjects more likely to be more severely cognitively challenged; and clinical pattern of cerebral palsy $(P=.001)$. In those subjects with scored imaging studies $(\mathrm{n}=128)$, higher periventricular leukomalacia scores were strongly associated with the presence of epilepsy $(P=.001)$. Significant associations were also found between epilepsy and cortical visual impairment and neonatal seizures.

Patient age was independently associated with epilepsy $(P=.028)$, with a mean age of 12.2 years among those with epilepsy and 10.2 years among those without. Our patient sample was a median age of 11 years (range, 1-19 years) at the time of chart abstraction. However, since age was defined relative to the date of chart abstraction (September 2000), rather than to the date of presentation, the variable's limited scientific value necessitated its exclusion from subsequent analyses.

Differences between the epileptic and nonepileptic groups sufficient to permit inclusion in the logistic regression analysis $\left(\chi^{2} P \leq .200\right)$ were found for 7 dichotomous factors (Table 1): a history of inability to walk independently, mental handicap (or an intelligence quotient of $<70$ ), cerebral palsy pattern other than spastic diparesis, a more severe grade of periventricular leukomalacia, cortical visual impairment, neonatal seizures, and term delivery.

The results of the logistic regression analysis are summarized in Table 2, which includes both unadjusted and adjusted odds ratios. Of the 7 factors analyzed, 6 did not yield statistically significant adjusted odds ratios $(P>.05)$, and only a history of neonatal seizures was strongly associated with epilepsy.

Post hoc $\chi^{2}$ and $t$ test analyses were performed to examine relationships between neonatal seizures and factors that might mediate an association with epilepsy: infection and bleeding during pregnancy, postnatal ventilation, 1-minute Apgar score, intraventricular hemorrhaging, and preterm delivery. No significant results were found. In our sample, $12(57.1 \%)$ of 21 subjects with neonatal seizures went on to develop epilepsy compared with 28 subjects $(21.1 \%)$ with epilepsy among the 133 who did not have neonatal seizures.

\section{Radiologic Findings}

Of the 154 subjects with documented periventricular leukomalacia, 17 had only cranial ultrasound imaging, and CT/MRI studies were unavailable for analysis in 9, leaving 128 subjects $(83.1 \%$ of those with confirmed periventricular leukomalacia) whose films were scored by the team of radiologic investigators. The incidence of epilepsy in the scored subjects was $28.9 \%(37 / 128)$, similar to that of the complete cohort.

Of the subjects with periventricular leukomalacia scores, $102(79 \%)$ of 128 had been studied by CT alone, and $26(20.3 \%)$ by MRI, either alone or with CT. Of the 128 evaluated subjects, 22 scored in category 1,58 in category 2,39 in category 3 , and 9 in category 4 . The incidences of epilepsy in the 4 groups were, respectively, $22.7 \%, 15.5 \%, 43.6 \%$, and $66.7 \%$. Thus, only 9 of 128 demonstrated pathology other than periventricular leukomalacia (ie, score category 4 ) of whom 6 were epileptic. All 9 subjects with score category 4 had their focal pathology detected by CT; MRI did not reveal pathology not already evident on CT. Thirty-one epileptic subjects appeared to have only periventricular leukomalacia, with 17 (45.9\%) having severe white matter injury (score category 3 ). The radiologic abnormalities identified in the score category 4 group included porencephalic cysts in 5, focal cortical atrophy in 2, small cortical/subcortical infarct in 1, and intracerebral calcification in 1 . All 5 of the subjects with porencephaly were reported to have had intracranial hemorrhages in the neonatal period. 
Table 1. The Association of Selected Factors With Epilepsy in Children With Periventricular Leukomalacia

\begin{tabular}{|c|c|c|c|}
\hline Putative Risk Factor For Epilepsy & No Epilepsy, n (\%) & Has Epilepsy, n (\%) & P (association with epilepsy) \\
\hline \multicolumn{4}{|l|}{ Categoric variables } \\
\hline Ambulatory status in 3 categories & $(\mathrm{n}=112)$ & $(n=40)$ & $.008^{\mathrm{a}}$ \\
\hline Independent walking & $57(50.9)$ & $15(37.5)$ & \\
\hline Walking with aid(s) & $38(33.9)$ & $8(20.0)$ & \\
\hline Wheelchair & $17(15.2)$ & $17(42.5)$ & \\
\hline Cognitive function in 4 categories & $(\mathrm{n}=114)$ & $(\mathrm{n}=40)$ & $<.001^{\mathrm{b}}$ \\
\hline Normal & $42(36.8)$ & $2(5.0)$ & \\
\hline Learning disabled & $26(22.8)$ & $12(30.0)$ & \\
\hline Mentally handicapped $(\mathrm{IQ}<70)$ & $33(28.9)$ & $22(55.0)$ & \\
\hline Too young or deaf & $13(11.4)$ & $4(10.0)$ & \\
\hline Clinical pattern of cerebral palsy in 5 categories & $(n=114)$ & $(n=40)$ & $.001^{\mathrm{b}}$ \\
\hline Spastic diparesis & $89(78.1)$ & $17(42.5)$ & \\
\hline Leg dominant quadriparesis & $18(15.8)$ & $15(37.5)$ & \\
\hline Triparesis & $4(3.5)$ & $5(12.5)$ & \\
\hline Mixed spastic diparesis or dystonic & $1(0.9)$ & $2(5.0)$ & \\
\hline Other & $2(1.8)$ & $1(2.5)$ & \\
\hline \multicolumn{4}{|l|}{ Dichotomous and collapsed variables } \\
\hline Ambulation & $(\mathrm{n}=114)$ & $(\mathrm{n}=40)$ & $.196^{\mathrm{c}}$ \\
\hline Independent walking & $57(50.9)$ & $15(37.5)$ & \\
\hline Other & $55(49.1)$ & $25(62.5)$ & \\
\hline Cognitive ability & $(\mathrm{n}=101)$ & $(\mathrm{n}=36)$ & $.005^{\mathrm{c}}$ \\
\hline Mental handicap & $33(32.7)$ & $22(61.1)$ & \\
\hline Other & $68(67.3)$ & $14(38.9)$ & \\
\hline Motor disability & $(\mathrm{n}=114)$ & $(\mathrm{n}=40)$ & $<.001^{\mathrm{c}}$ \\
\hline Spastic diparesis & $89(78.1)$ & $17(42.5)$ & \\
\hline Other & $25(21.9)$ & $23(57.5)$ & \\
\hline Periventricular leukomalacia severity & $(\mathrm{n}=91)$ & $(\mathrm{n}=37)$ & $<.001^{\mathrm{c}}$ \\
\hline Categories 3 and 4 & $25(27.5)$ & $23(62.2)$ & \\
\hline Categories 1 and 2 & $66(72.5)$ & $14(37.8)$ & \\
\hline Cortical visual impairment & $(\mathrm{n}=114)$ & $(\mathrm{n}=39)$ & $<.001^{\mathrm{c}}$ \\
\hline No & $107(93.9)$ & $25(64.1)$ & \\
\hline Yes & $7(6.1)$ & $14(35.9)$ & \\
\hline History of neonatal seizure & $(\mathrm{n}=114)$ & $(\mathrm{n}=40)$ & $.001^{\mathrm{c}}$ \\
\hline No & $105(92.1)$ & $28(70.0)$ & \\
\hline Yes & $9(7.9)$ & $12(30.0)$ & \\
\hline Intrauterine growth retardation & $(\mathrm{n}=114)$ & $(\mathrm{n}=40)$ & $.511^{\mathrm{c}}$ \\
\hline No & $106(93.0)$ & $36(90.0)$ & \\
\hline Yes & $8(7.0)$ & $4(10.0)$ & \\
\hline Sex & $(\mathrm{n}=114)$ & $(\mathrm{n}=40)$ & $1.000^{\mathrm{c}}$ \\
\hline Male & $65(57.0)$ & $23(57.5)$ & \\
\hline Female & $49(43.0)$ & $17(42.5)$ & \\
\hline Bleeding during pregnancy & $(\mathrm{n}=110)$ & $(n=40)$ & $.395^{\mathrm{c}}$ \\
\hline No & $85(77.3)$ & $28(70.0)$ & \\
\hline Yes & $25(22.7)$ & $12(30.0)$ & \\
\hline Preterm delivery & $(\mathrm{n}=114)$ & $(n=40)$ & $.178^{\mathrm{c}}$ \\
\hline No & $20(17.5)$ & $11(27.5)$ & \\
\hline Yes & $94(82.5)$ & $29(72.5)$ & \\
\hline Intraventricular hemorrhaging & $(\mathrm{n}=114)$ & $(\mathrm{n}=40)$ & $.314^{\mathrm{c}}$ \\
\hline No & $84(73.7)$ & $26(65.0)$ & \\
\hline Yes & $30(26.3)$ & $14(35.0)$ & \\
\hline Family history of epilepsy & $(\mathrm{n}=107)$ & $(\mathrm{n}=40)$ & $.325^{\mathrm{c}}$ \\
\hline No & $91(85.0)$ & $31(77.5)$ & \\
\hline Yes & $16(15.0)$ & $9(22.5)$ & \\
\hline Infection during pregnancy & $(\mathrm{n}=110)$ & $(\mathrm{n}=40)$ & $1.000^{\mathrm{c}}$ \\
\hline No & $95(86.4)$ & $35(87.5)$ & \\
\hline Yes & $15(13.6)$ & $5(12.5)$ & \\
\hline
\end{tabular}

NOTE: $I Q=$ intelligence quotient.

a. Mann-Whitney $U$ test.

b. Fisher exact test.

c. $\chi^{2}$ test. 
Table 2. Adjusted and Unadjusted Factors Associated With Epilepsy in Children With Periventricular Leukomalacia

\begin{tabular}{lcc}
\hline $\begin{array}{l}\text { Risk Factor Associated } \\
\text { Wth Epilepsy }\end{array}$ & $\begin{array}{c}\text { Unadjusted } \\
\text { OR }\end{array}$ & $\begin{array}{c}\text { Adjusted } \\
\text { OR }(95 \% \mathrm{CI})\end{array}$ \\
\hline $\begin{array}{l}\text { PVL grade (more severe } \\
\text { vs less severe) }\end{array}$ & 3.9 & $2.6(0.9,7.5)$ \\
$\begin{array}{c}\text { Motor disability (spastic } \\
\text { diparesis vs other types) }\end{array}$ & 0.2 & $0.4(0.1,1.4)$ \\
$\begin{array}{c}\text { Cortical visual impairment } \\
\quad \text { present vs absent) }\end{array}$ & 8.6 & $2.6(0.6,11.1)$ \\
$\begin{array}{c}\text { Ambulation (independent } \\
\text { walking vs other types) }\end{array}$ & 0.6 & $1.5(0.5,4.9)$ \\
$\begin{array}{c}\text { Cognitive ability (mentally } \\
\text { disabled or IQ }<70\end{array}$ & 3.2 & $1.2(0.4,3.8)$ \\
$\begin{array}{c}\text { vs others) } \\
\text { Preterm delivery } \\
\quad \text { preterm vs term) }\end{array}$ & 0.6 & $0.5(0.2,1.6)$ \\
$\begin{array}{c}\text { Neonatal seizure } \\
\quad(\text { present vs absent) }\end{array}$ & 5 & $5.1(1.4,17.7)$ \\
\hline
\end{tabular}

NOTE: $\mathrm{OR}=$ odds ratio; $\mathrm{CI}=$ confidence interval $\mathrm{PVL}=$ periventricular leukomalacia; $\mathrm{IQ}=$ intelligence quotient.

\section{Discussion}

To our knowledge, we are reporting data concerning epilepsy in the first large-scale study of cerebral palsy patients with a specific predominant cerebral pathology, radiologically defined periventricular leukomalacia, rather than a mixture of all types of cerebral palsy and associated pathology.

In our study, the diagnosis of periventricular leukomalacia was based on the characteristic radiologic picture of bilateral cerebral white matter atrophy with apparent cortical sparing in the context of a clinical picture of spastic cerebral palsy. Other forms of static white matter pathology could, in principle, produce similar radiologic findings, but it is unlikely that the associated clinical picture in such cases would fit our clinical inclusion criteria. Primary central white matter hypoplasia syndromes produce a radiologic picture of reduced cerebral white matter volume and absence of cerebral hemispheric myelination. ${ }^{18}$ Clinically, however, such patients are typically hypotonic rather than spastic and are severely mentally handicapped. ${ }^{18}$ Periventricular hemorrhagic infarction also leads to periventricular white matter atrophy with cortical sparing, but the pathology is typically unilateral and the patients have hemiparesis. ${ }^{19} \mathrm{~A}$ recent report suggests that periventricular hemorrhagic infarction is occasionally bilateral but, if so, is invariably asymmetric. ${ }^{20} \mathrm{In}$ the light of this information, we cannot exclude the possibility that a few of our patients with grade 4 intraventricular hemorrhage may have had bilateral periventricular hemorrhagic infarction rather than periventricular leukomalacia.

Current evidence suggests that the radiologic appearance of periventricular leukomalacia may be the result of 2 distinct forms of white matter pathology resulting from ischemic or cytokine-mediated mechanisms in premature infants: focal cystic periventricular leukomalacia and diffuse white matter injury and hypomyelination. ${ }^{21}$ The latter pathology results from injury to preoligodendrocytes. ${ }^{22}$ Because the 2 pathologies have similar etiologies and are associated with spastic diparesis and leg-dominant quadriparesis, they continue to be combined under the umbrella term of periventricular leukomalacia. ${ }^{23}$

Two of the previously reported series of patients with cerebral palsy and periventricular leukomalacia ${ }^{1-9}$ have included cohorts with periventricular leukomalacia detected through routine imaging studies of the whole cerebral palsy population. Carlsson et $\mathrm{al}^{6}$ identified 34 subjects with periventricular leukomalacia from a population base of 600000 ; it is not clear from their report whether any of their cohort had any other radiologic findings. Okumura et $\mathrm{al}^{9}$ found 80 subjects with isolated periventricular leukomalacia in a population base of 700000 , and there were also a small, unidentified number of subjects with periventricular leukomalacia plus porencephaly.

The only study specifically dealing with epilepsy in cerebral palsy patients with periventricular leukomalacia was that of Gurses et al. ${ }^{13}$ This study, however, was partly based in a tertiary referral center for epilepsy, was not populationbased, and dealt with small patient numbers ( 2 cohorts of 19 and 12, respectively, the latter all epileptic).

Although the above studies all consider, variously, some risk factors for epilepsy (eg, clinical pattern, severity, radiologic findings), none reports a systematic analysis of a large number of potentially relevant factors. The 1 study of cerebral palsy and epilepsy reporting a logistic regression analysis $^{7}$ dealt with a mixed population of 198 cases from a catchment area of 1.2 million. Because we identified 215 cerebral palsy subjects just having clinical patterns associated with periventricular leukomalacia, also based on a population of 1.2 million, it seems unlikely that the study of Kulak and Sobaniec ${ }^{7}$ dealt with all children with cerebral palsy in the area, perhaps because the authors were based in a tertiary referral center.

Of the 198 cerebral palsy cases in the Kulak and Sobaniec study, 26 of 192 were identified as having periventricular leukomalacia on CT, and $14(53.8 \%)$ of the 26 were epileptic, an epilepsy incidence similar to the $47.4 \%$ of Gurses et $\mathrm{al}^{13}$ but much higher than our incidence figure and the $23.5 \%$ reported by Carlsson et $\mathrm{al}^{6}$ and the $16.0 \%$ by Okumura et al, ${ }^{9}$ all based on larger patient numbers. Thus, our logistic regression analysis has the advantage of being population-based and devoted specifically to 1 type of cerebral palsy pathology.

In our risk factor analysis of periventricular leukomalacia subjects, we found an apparent correlation between epilepsy and spastic quadriparesis, severe cognitive impairment, lack of independent ambulation, cortical visual impairment, term delivery, neonatal seizures, and radiologic severity. After correction for potentially confounding 
variables, however, we found that the only variable still strongly associated with the development of epilepsy was a history of neonatal seizures.

In this respect, our findings differ from those of Kulak and Sobaniec, ${ }^{7}$ who, after logistic regression analysis, also found a significant correlation between epilepsy and low birth weight, family history of epilepsy, cerebral palsy severity, and severity of CT abnormalities. These differences likely stem from the difference in cerebral palsy populations evaluated. Because our univariate analysis also showed a significant correlation between epilepsy and both cerebral palsy and periventricular leukomalacia severity, it is likely that severity of pathology is a contributing factor to the development of epilepsy. Nevertheless, with specific reference to periventricular leukomalacia, other factors may be more important.

Our study's finding of a strong adjusted association between epilepsy in periventricular leukomalacia and a history of neonatal seizures reinforces the same correlation for all types of cerebral palsy reported by Carlsson et al, ${ }^{6}$ Kulak and Sobaniec, ${ }^{7}$ and Gururaj et al. ${ }^{8}$ Seizures in the immature brain are known to induce neuronal death, although neonates can tolerate relatively prolonged seizures without suffering massive cell death, possibly owing to low cerebral metabolic rate and fragmentary neuronal networks. ${ }^{24}$ Although experimental studies have found that seizures early in life have little consequence on brain development, more recent animal models suggest that such events may have long-term clinically relevant consequences, such as spatial learning deficits. ${ }^{25}$

Why neonatal seizures predispose individuals with periventricular leukomalacia to the subsequent development of epilepsy is open to speculation. It is possible that sick neonates are more likely to seize if there is acute cortical damage in addition to periventricular leukomalacia. If so, the cortical pathology would have to be of sufficiently small volume as not to be visible on subsequent cerebral imaging: only 3 of our subjects with neonatal seizures had later radiologic evidence of focal cortical atrophy or infarction.

A second possibility is the presence of acute intracerebral and intraventricular bleeding, as iron has been shown to be potentially epileptogenic in animal models. ${ }^{26,27}$ Our sample, however, failed to show a statistically significant difference in intraventricular bleeding between subjects with neonatal seizures versus those without. Infection and maternal bleeding during pregnancy were also found to be insignificant with respect to neonatal seizures. Perinatal hypoxia has been variably associated with the development of epilepsy in later life, ${ }^{28}$ but we found no independent association between a history of neonatal seizures and preterm birth, 1-minute Apgar score, or requirement for ventilatory support in the neonatal period. Thus, hypoxia cannot be confirmed as the most likely avenue between neonatal seizures and eventual epilepsy.
According to Mizrahi et al, ${ }^{29}$ however, the quality of the seizures in neonates is the main determinant of longterm outcome; prognosis varies with the etiology of the neonatal seizure, ${ }^{29}$ an aspect not measured in this study. As suggested by our results, and by evidence that neonatal seizures are also associated with a number of activitydependent changes in brain development (eg, altered synaptogenesis and reduction in neurogenesis), ${ }^{30}$ neonatal seizures should not be considered benign events.

With respect to possible radiologic correlates with epilepsy, Gurses et $\mathrm{al}^{13}$ reported the presence of cortical abnormalities on MRI in 2 cohorts of periventricular leukomalacia subjects: 19 from 1 center (9 with epilepsy), and 12 from a second center (all with epilepsy). Four of 9 epileptic periventricular leukomalacia subjects from the first center and 7 of 12 from the second center were reported to have cortical abnormalities on MRI. The nature of the cortical abnormalities was not specified except for a single subject described as having occipital cortical atrophy.

In contrast, we did not find focal radiologic pathology to occur frequently in epileptic subjects with periventricular leukomalacia $(6 / 37,16.2 \%)$. Most epileptic subjects (83.8\%) demonstrated only periventricular leukomalacia, typically of a more severe degree $(24.3 \%$ score category $2,45.9 \%$ category 3$)$. This finding raises the possibility that white matter damage itself might be potentially epileptogenic.

In experimental animals, there is some support for this hypothesis. If cerebral cortex is anatomically isolated by means of disconnection from white matter (the so-called chronic isolated cortex), it becomes epileptogenic. ${ }^{31-33}$ Epileptic seizures are also occasionally reported in patients with multiple sclerosis who have large subcortical demyelinated plaques, particularly in the temporal lobe. ${ }^{34}$

This study has some potential weaknesses, which largely stem from its retrospective nature. Our study was population-based rather than originating in a tertiary referral center, but it is possible that our periventricular leukomalacia cohort is not entirely representative of the spectrum of patients with this pathology. Because we did not evaluate cerebral palsy patients with clinical patterns not known to be associated with periventricular leukomalacia (eg, arm-dominant quadriparesis, dyskinetic/dystonic quadriparesis), we may have missed a few cases with coincidental periventricular leukomalacia not apparent clinically. At the same time, the 61 additional cases lacking radiologic confirmation of periventricular leukomalacia could have, if included, modified our results.

Cortical pathology not readily visualized on CT or MRI remains a possible etiology for epilepsy not satisfactorily addressed by our study. Given that only $20.3 \%$ of the subjects in this retrospective analysis had received MRI studies, it is possible that minor focal cortical pathology was present in some of those who had only CT studies and was not detected. In addition, based on MRI volumetric data, periventricular leukomalacia may be 
associated with a diffuse reduction in cortical gray matter volume not appreciated on standard MR images. ${ }^{35}$

\section{Conclusion}

The significant adjusted association between neonatal seizures and the later development of epilepsy in cerebral palsy patients with periventricular leukomalacia (as distinct from all forms of cerebral palsy) reinforces the importance of neonatal seizures as a contributing cause of epilepsy in cerebral palsy and is a finding that warrants further investigation.

\section{Acknowledgment}

This work was presented in part at the Child Neurology Society meeting, Ottawa, Canada, October 2004.

\section{References}

1. Krägeloh-Mann I, Hagberg G, Meisner C, et al. Bilateral spastic cerebral palsy-a comparative study between south-west Germany and western Sweden. I. Clinical patterns and disabilities. Dev Med Child Neurol. 1993;35:1037-1047.

2. Hadjipanayis A, Hadjichristodoulu C, Youroukos S. Epilepsy in patients with cerebral palsy. Dev Med Child Neurol. 1997;39: 659-663.

3. Kwong KL, Wong SN, So KT. Epilepsy in children with cerebral palsy. Pediatr Neurol. 1998;19:31-36.

4. Şenbil N, Sonel B, Aydin OF, Gürer YKY. Epileptic and nonepileptic cerebral palsy: EEG and cranial imaging findings. Brain Dev. 2002;24:166-169.

5. Singhi P, Jagirdar S, Khandelwal N, Malhi P. Epilepsy in children with cerebral palsy. J Child Neurol. 2003;18:174-179.

6. Carlsson M, Hagberg G, Olsson I. Clinical and aetiological aspects of epilepsy in children with cerebral palsy. Dev Med Child Neurol. 2003;45:371-376.

7. Kulak W and Sobaniec W. Risk factors and prognosis of epilepsy in children with cerebral palsy in north-eastern Poland. Brain Dev. 2003;25:499-506.

8. Gururaj AK, Sztriha L, Bener A, et al. Epilepsy in children with cerebral palsy. Seizure. 2003;12:110-114.

9. Okumura A, Hayakawa F, Kato T, et al. Epilepsy in patients with spastic cerebral palsy: correlation with MRI findings at 5 years of age. Brain Dev. 1999;21:540-543.

10. Okumura A, Hayakawa F, Kuno K, Watanabe K. Periventricular leukomalacia and West syndrome. Dev Med Child Neurol. 1996; 38:13-18.

11. Hagberg B, Hagberg G. The origins of cerebral palsy. Rec Adv Ped. 1993;11:67-83.

12. Humphreys $P$, Whiting S, Pham B. Hemiparetic cerebral palsy: clinical pattern and imaging in prediction of outcome. Can J Neurol Sci. 2000;27:210-219.

13. Gurses C, Gross DW, Andermann F, et al. Periventricular leukomalacia and epilepsy. Incidence and seizure pattern. Neurology. 1999;52:341-345.

14. Ozawa H, Hashimoto T, Endo T, et al. West syndrome with periventricular leukomalacia: a morphometric MRI study. Pediatr Neurol. 1998;19:358-363.
15. Flodmark O, Roland EH, Hill A, Whitfield MF. Periventricular leukomalacia: radiological diagnosis. Radiology. 1987;162: 119-124.

16. Grant PE, Barkovich AJ. Neuroimaging in CP: issues in pathogenesis and diagnosis. Mental Retardation and Devel Disabilities. 1997;3:118-128.

17. Walter SD, Eliaziw M, Donner A. Sample size and optimal designs for feasibility studies. Studies in Medicine. 1998;17:101-110.

18. Diamond AL, Kollmeyer CA, Bicknese AR. Primary central white matter hypoplasia of the neocortex. Pediatr Neurol. 2005; 32:40-45.

19. Takanashi J, Barkovich AJ, Ferriero DM, et al. Widening spectrum of congenital hemiplegia: periventricular venous infarction in term neonates. Neurology. 2003;61:531-533.

20. Bassan H, Benson CB, Limperopoulos C, et al. Ultrasonographic features and severity scoring of periventricular hemorrhagic infarction in relation to risk factors and outcome. Pediatrics. 2006; 117:2111-2118.

21. Black SA, Rivkees SA. Emerging concepts in periventricular white matter injury. Semin Perinatol. 2004;28:405-414.

22. Counsell SJ, Allsop JM, Harrison MC, et al. Diffusion-weighted imaging of the brain in preterm infants with focal and diffuse white matter abnormality. Pediatrics. 2003;112:1-7.

23. Volpe JJ. Cerebral white matter injury of the premature infant-more common than you think. Pediatrics. 2003;112: 176-180.

24. Wasterlain CG, Niquet J, Thompson KW, et al. Seizure-induced neuronal death in the immature brain. Prog Brain Res. 2002; 135:335-353.

25. Swann JW. Recent experimental studies of the effects of seizures on brain development. Progress In Brain Research. 2002;135:391-393.

26. Willmore LJ, Sypert GW, Munson JV, Hurd RW. Chronic focal epileptiform discharges induced by injection of iron into the rat and cat cortex. Science. 1978;200:1501-1503.

27. Willmore LJ, Rubin JJ. Effects of antiperoxidants on FeCl2induced lipid peroxidation and focal edema in rat brain. Exp Neurol. 1984;83:62-70.

28. Bergamasco B, Penna P, Ferrero P, Gavinelli R. Neonatal hypoxia and epileptic risk: a clinical prospective study. Epilepsia. 1984; 25:131-146.

29. Mizrahi EM, Clancy RR. Neonatal seizures: early-onset seizure syndromes and their consequences for development. Ment Retard Dev Disabil Res Rev. 2000;6:229-241.

30. Holmes GL, Khazipov R, Ben-Ari Y. New concepts in neonatal seizures. Neuroreport. 2002;13:A3-A8.

31. Echlin F, Battista J. Epileptiform seizures from chronic isolated cortex. Arch Neurol. 1963;9:154-170.

32. Sharpless S, Halpern L. The electrical excitability of chronically isolated cortex studies by means of chronically implanted electrodes. Electroencephalogr Clin Neurophysiol. 1962;14:244-255.

33. Prince DA, Jacobs K. Inhibitory function in two models of chronic epileptogenesis. Epilepsy Res. 1998;32:83-92.

34. Gambardella A, Valentino P, Labate A, et al. Temporal lobe epilepsy as a unique manifestation of multiple sclerosis. Can J Neurol Sci. 2003;30:228-232.

35. Inder TE, Huppi PS, Warfield S, et al. Periventricular white matter injury in the premature infant is followed by reduced cerebral cortical gray matter volume at term. Ann Neurol. 1999; 46:755-760. 